\title{
Performance Enhancement of Optical CDMA by Differential-Phase Method for Radio-over-Fiber Transmissions
}

\author{
Hsu-Chih Cheng, ${ }^{1}$ Chih-Ta Yen, ${ }^{2}$ and Ing-Jr Ding ${ }^{2}$ \\ ${ }^{1}$ Department of Electro-Optical Engineering, National Formosa University, Yunlin County 632, Taiwan \\ ${ }^{2}$ Department of Electrical Engineering, National Formosa University, Yunlin County 632, Taiwan
}

Correspondence should be addressed to Chih-Ta Yen; chihtayen@gmail.com

Received 8 September 2013; Accepted 7 October 2013

Academic Editor: Teen-Hang Meen

Copyright (C) 2013 Hsu-Chih Cheng et al. This is an open access article distributed under the Creative Commons Attribution License, which permits unrestricted use, distribution, and reproduction in any medium, provided the original work is properly cited.

\begin{abstract}
The study proposes the differential-phase optical code-division multiple-access (OCDMA) network for radio-over-fiber (RoF) transmissions, and the characteristics are numerically analyzed. The network coder/decoders (codecs) are structured on the basis of arrayed-waveguide-grating (AWG) routers with complementary Walsh-Hadamard (CWH) signature codes. In the proposed system, the network requires only two AWG routers to accomplish spectral encoding of radio base station (RBS) and decoding of control station for the complementary keying, thus resulting in a simpler and low cost system. Performance analyses are evaluated with the dominant noise of phase-induced intensity noise (PIIN) in spectral code OCDMA network. By the proposed AWG-based OCDMA with the differential-phase scheme, it is possible to establish interference-free and low crosstalk beat noise RoF systems.
\end{abstract}

\section{Introduction}

The millimeter-wave radio-over-fiber (RoF) system has drawn much attention on the realization of broadband radio access services recently. This is because of RoF technology can resolve the scarcity of available radio frequency $(\mathrm{RF})$ resource problem [1-5]. It will become an important network access scheme other than the fiber-to-the-home (FTTH) technology. As a configuration of RoF network, microcells are connected by optical fibers among radio base stations (RBS) and central control station (CS).

Optical code-division multiple-access (OCDMA) is one candidate scheme for RoF network access technique that allows multiple users in local area networks (LANs) to access the same fiber channel asynchronously without delay or scheduling. This property is also powerful for RoF access because of its asynchronous access, flexibility, and transparency for various radio air interfaces.

In general, traditional incoherent OCDMA uses unipolar sequences as signature codes, and the coded method is usually based on the time domain. However, it is a problem to suppress multiple-access interference (MAI), and the code length of the codes is always long to support simultaneous users in the systems. In the recent researches, spectral amplitude coding (SAC) scheme [4-11] of OCDMA becomes more popular because of the MAI-elimination and low cost set up components of incoherent optical sources and optical filter in the system. There are many code families that can be used in SAC-OCDMA network such as maximal-length sequence (M-sequence) codes [8], Walsh-Hadamard codes [9-11], modified quadratic congruence (MQC) codes [6], and modified PN codes [4].

The SAC-OCDMA network codecs can be constructed with fiber Bragg grating (FBG) devices $[6,7]$, but the physical size of FBG arrays will become impractical when the number of network users becomes large. The other kind of implementation option uses arrayed waveguide grating (AWG) router as codec on OCDMA network [11]. It needs mirrors and circulators to code the data, which loses the power and increases the system cost. The two-dimensional (2D) wavelength/time spreading OCDMA system in [12] implements multidimensional codes utilizing AWG multiplexers and fiber delay lines. This scheme is limited by the multiple-access interference (MAI) and is not easy to be implemented for analog RoF network. The other kind of digital OCDMA network [8] with AWG codec is proposed. 


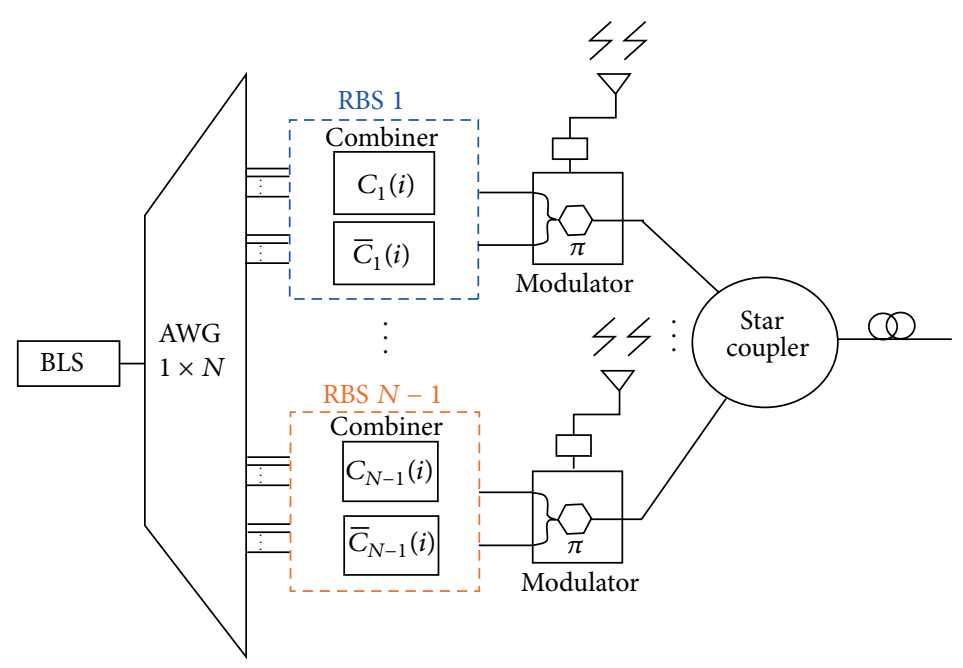

FIGURE 1: The proposed RBS transmitters and AWG encoders.

Unfortunately, it will increase the crosstalk beat noise $[13,14]$ when the number of active users becomes large. In spectrumbased coding OCDMA system, the number of simultaneous active users is limited to the beat noise or calls phaseinduced intensity noise (PIIN). It accumulated at balanced photodetector (PD) during decoding process. In this study, we propose the interference-free RoF system structured with AWG routers [15], differential-phase intensity modulators coded with complementary Walsh-Hadamard (CWH) codes in the transmitter and balanced photodetector scheme in the receiver. The scheme can reduce the physical size of the coder and is also constructed without any sampling technique and aliasing canceller. The carrier-to-noise (CNR) ratio of proposed scheme with CWH code is superior to conventional SAC with M-sequence and Walsh-Hadamard code about $7.7 \mathrm{~dB}$ in RoF system.

The remainder of this paper is organized as follows. In Section 2, spectral encoding scheme using $\mathrm{CWH}$ code is described. In Section 3, the system encoder and decoder are presented. Section 4 evaluates performance of the proposed system in terms of CNR ratio and bit-error-rate conditions for PIIN. Finally, conclusions are presented in Section 5.

\section{Spectrum Coding Scheme with the Complement Walsh-Hadamard Code}

The unipolar Walsh-Hadamard code sequences $\mathbf{C}_{k}=\left(c_{k}(0)\right.$, $\left.c_{k}(1), \ldots, c_{k}(N-1)\right)$ are $(0,1)$ sequences of length $N$ assigned as the signature code for RBS $k$, where $k \in\{0,1, \ldots, N-1\}$, and the periodic correlation between $\mathbf{C}_{k}$ and $\mathbf{C}_{j}$ is defined as

$$
\mathrm{R}_{c c}(k, j)=\sum_{i=0}^{N-1} c_{k}(i) c_{j}(i) .
$$

We assume that complementary spectral $\overline{\mathbf{C}}_{k}=\left(\bar{c}_{k}(0)\right.$, $\left.\bar{c}_{k}(1), \ldots, \bar{c}_{k}(N-1)\right)$, where $\overline{\mathbf{C}}_{k}(i)=1-\mathbf{C}_{k}(i)$ for $i=$
$0,1, \ldots, N-1$. The periodic correlation between $\overline{\mathbf{C}}_{k}$ and $\mathbf{C}_{j}$ can be expressed as

$$
R_{\bar{C} C}(k, l)=\sum_{i=0}^{N-1} \bar{c}_{k}(i) c_{j}(i) .
$$

According to the property of code families that $R_{C C}(k, j)-$ $R_{\bar{C} C}(k, j)=0$ for $k \neq j$, that can reject the influence of MAI coming from other RBSs.

In the proposed differential-phase scheme using $\mathrm{CWH}$ code, the RBS $k$ will send codeword $\mathrm{C}_{k}$ (for in-phase radio signal $k$ ) and its complement codeword $\overline{\mathbf{C}}_{k}$ (for out-of-phase radio signal $k$ ) at the same time. Thus by combining the orthogonal property of $\mathbf{C}_{k}$ and $\overline{\mathbf{C}}_{k}$ codes to get phase diversity of radio signal, CNR will increase approximately $7.7 \mathrm{~dB}$ comparing with traditional coding single phase technologies. Each RBS requires only two AWG router and combiners to implement spectral encoding and decoding, respectively. Therefore, the fiber radio system using AWG router with CWH codes can be realized.

\section{System Description}

The proposed SAC-OCDMA system utilizes broadband light sources (BLSs) and AWG routers. Complementary keying is employed for each radio signal by directing the light from incoherent sources to input port of AWG encoder, and then AWG router output ports are connected to combiners according to codeword $\mathbf{C}_{k}$ and its complement codeword $\overline{\mathbf{C}}_{k}$ to generate the amplitude spectrum of transmitted radio signal.

Figure 1 shows the proposed differential-phase transmitters and AWG encoders. In each RBS transmitter, differentialphase intensity modulator is performed when the radio signal of each RBS is used to analog or digital modulation. A BLS spectrum is filtered for one free spectral range (FSR) of the AWG router. The codeword $\mathbf{C}_{k}$ which takes the in-phase radio signal $k$ and $\overline{\mathbf{C}}_{k}$ which takes the out-of-phase radio 


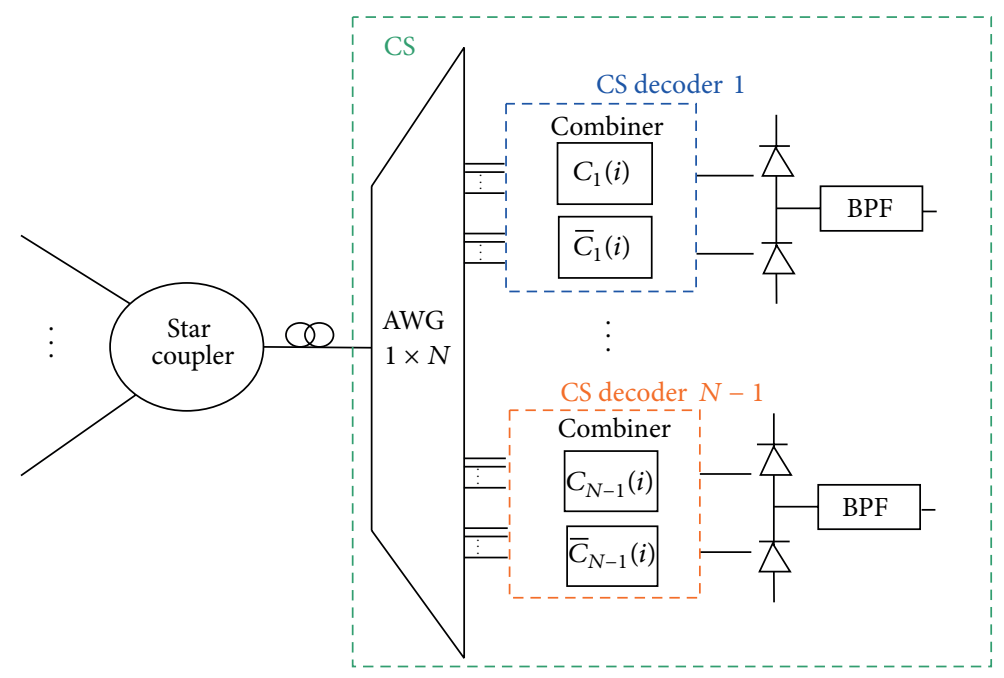

FIgURE 2: The proposed CS receiver and AWG decoders.

signal $k$ will be transmitted when the BLS is directed into the first input port of the AWG router in the transmitter $k$. The advantage of complementary encoding is accomplished with only one AWG router for each RBS.

After the encoding process, the coded spectra of $\mathbf{C}_{k}$ and $\overline{\mathbf{C}}_{k}$ become the optical carriers to take the in-phase radio signal and out-of-phase radio signal simultaneously via differential-phase intensity modulator. All coded optical signals of each RBS then collected by the star coupler and broadcasted to the CS.

The radio signal $r_{k}(t)$ at the transmitter has the form

$$
r_{k}(t)=a_{k}(t) \cos \left(2 \pi f_{r f} t\right)
$$

where $f_{r f}$ is the carrier frequency of the radio signal and $a_{k}(t)$ is the complex envelope with a bandwidth $B_{r f}$.

The received optical signal spectrum $\mathbf{S}$ is the summation of all RBSs' transmitted signal spectrum as follows:

$$
\begin{aligned}
\mathbf{S} & =\left(s_{0}, s_{1}, \ldots, s_{N-1}\right) \\
& =\sum_{k=0}^{N-1}\left\{\left[1+m r_{k}(t)\right] \mathbf{C}_{k}+\left[1-m r_{k}(t)\right] \overline{\mathbf{C}}_{k}\right\},
\end{aligned}
$$

where $m$ is modulation index and $r_{k}(t)$ is the $k$ th RBS's radio signal.

The AWG router-based decoders are shown in Figure 2. The star coupler is connected to the decoder's AWG router, which distributes received signals to the balanced PDs of each CS decoder to realize differential decoding process. Taking CS decoder $k$ as the example, connections from output ports of the AWG router to combiners are determined by the $\mathbf{C}_{k}$ codeword and its complement codeword $\overline{\mathbf{C}}_{k}$. The received signal $\mathbf{S}$ coming from the star coupler is connected to the AWG router directly; its can reduce the loss by using the split device and can also suppress the crosstalk beat noise of AWG router [8] because it is only connected to one input port. The balanced $\mathrm{PD}$ of $\mathrm{RBS} k$ will receive $\mathrm{SC}_{k}$ in the upper arm of $\mathrm{PD}$ and $\overline{\mathbf{S C}}_{k}$ in the lower arm of $\mathrm{PD}$. After correlation subtraction of $\mathbf{S C}_{k}-\mathbf{S} \overline{\mathbf{C}}_{k}$ is performed in the balanced $\mathrm{PD}$, the radio signal for the RBS $k$ will be extracted, and other RBSs' interferences are rejected.

\section{Performance Analysis and Discussion}

The performance of the proposed 2D OCDMA system is limited by shot, thermal, and PIIN noises, particularly when the received power is high. PIIN results from the beating of incoherent light fields during the direct detection of squarelaw PDs, and its magnitude depends essentially on the state of polarizations (SOPs) and spectra of the optical signals. To simplify the current system performance analysis, the following assumptions are made.

(i) The power spectral density (PSD) of each light source is ideally flat over the optical bandwidth $\nu_{o}+\Delta \nu / 2$, where $v_{o}$ is the central optical frequency and $\Delta v$ is the common optical source bandwidth in hertz.

(ii) Sufficient chip time width delays exist between the arrivals of successive pulses.

(iii) Each coding chip has an identical spectral width of $\Delta v / N$.

(iv) The chip streams from each RBS are synchronous.

(v) $T_{F}$ is set equal to $1 /\left(2 B_{r f}\right)$.

(vi) The transmitted radio signal that is set to the timeaverage power $\left\langle r_{k}^{2}(t)\right\rangle$ equals-one half (i.e., $\left\langle r_{k}^{2}(t)\right\rangle=$ $1 / 2)$.

The light source spectrum of each RBS is assumed to be unpolarized and ideal flat over a bandwidth $\Delta v \mathrm{~Hz}$ with magnitude $P_{s r} / \Delta \nu$, where $P_{s r}$ is the effective power from a 
single source at the receiver. The instantaneous PSD of the received optical signals at star coupler can be written as

$$
\begin{aligned}
& s(\nu, t) \\
& =\frac{P_{s r}}{\Delta \nu} \sum_{k=1}^{K} \sum_{i=1}^{N}\left\{\left[1+m r_{k}(t)\right] \mathbf{C}_{k}(i)+[1-m(t)] \overline{\mathbf{C}}_{k}(i)\right\} \Pi(i),
\end{aligned}
$$

where

$$
\begin{aligned}
\Pi(i)= & u\left[v-v_{0}-\frac{\Delta v}{2 N}(-N+2 i-2)\right] \\
& -u\left[v-v_{0}-\frac{\Delta v}{2 N}(-N+2 i)\right],
\end{aligned}
$$

and $u(v)$ is the unit step function.

The instantaneous PSD of the upper PD and lower PD for CS decoder $l$ can be written as

$$
\begin{aligned}
& G_{1}(\nu, t) \\
& =\frac{P_{s r}}{\Delta \nu} \sum_{k=1}^{K} \sum_{i=1}^{N}\left\{\left[1+r_{k}(t)\right] \mathbf{C}_{k}(i)+\left[1-r_{k}(t)\right] \overline{\mathbf{C}}_{k}(i)\right\} \\
& \quad \times C_{l}(i) \Pi(i), \\
& G_{2}(\nu, t) \\
& =\frac{P_{s r}}{\Delta \nu} \sum_{k=1}^{K} \sum_{i=1}^{N}\left\{\left[1+r_{k}(t)\right] \mathbf{C}_{k}(i)+\left[1-r_{k}(t)\right] \overline{\mathbf{C}}_{k}(i)\right\} \\
& \quad \times \bar{C}_{l}(i) \Pi(i) .
\end{aligned}
$$

In (7), $G(\nu, t)$ is assumed to be the single sideband instantaneous PSD of the source.

The input current to the BPF can be written as

$$
\begin{aligned}
i_{o}(t)= & i_{o}(t)-i_{1}(t) i_{S_{k, l}}(t) \\
& +i_{\mathrm{MAI}}(t)+i_{\mathrm{PIIN}}(t)+i_{\text {shot }}(t) \\
& +i_{\text {thermal }}(t)
\end{aligned}
$$

where $i_{S_{k, l}}(t), i_{\mathrm{MAI}}(t), i_{\mathrm{PIIN}}(t), i_{\text {shot }}(t)$, and $i_{\text {thermal }}(t)$ are the desired signal, the interference, the PIIN noise, the shot noise, and the thermal noise, respectively.

In the proposed RoF system, differential-phase intensity modulator is employed to suppress the noise of nonlinearity during optical-electrical conversion process. We consider the worst case of proposed system when all RBSs transmit the maximum radio power, and it will cause the most noise power of PIIN. The PIIN power now becomes the dominant noise in our proposed RoF system.

To simplify the calculation, the radio signals are assumed as to be nonmodulated carries which have the autocorrelations function $R_{r_{k}}(\tau)$ and are expressed as

$$
R_{r_{k}}(\tau)=\frac{1}{2} \cos (2 \pi f \tau) .
$$

The variation of the photocurrent caused as a result of PIIN is given by:

$$
\left\langle I_{\text {PIIN }}^{2}\right\rangle=E\left[I^{2}\left(1+P^{2}\right) B \tau_{c}\right]
$$

where $E$ is the expectation operator, $I$ is the average photocurrent, $B$ is the noise-equivalent electrical bandwidth of the receiver, and $\tau_{c}$ is the coherence time of the source expressed as

$$
\tau_{c}=E\left\{\frac{\int_{0}^{\infty} G^{2}(\nu, t) d \nu}{\left[\int_{0}^{\infty} G(\nu, t) d \nu\right]^{2}}\right\}
$$

and the degree of polarization (DOP), $P$, is defined as

$$
P^{2}=\frac{\left(\left\langle s_{1}\right\rangle^{2}+\left\langle s_{2}\right\rangle^{2}+\left\langle s_{3}\right\rangle^{2}\right)}{\left\langle s_{0}\right\rangle^{2}}
$$

where $s_{0}, s_{1}, s_{2}$, and $s_{3}$ are Stoke parameters used to express the state of polarization (SOP). The bracket $\langle\cdot\rangle$ in (12) denotes the average value of the parameter over wavelength, time, or space. It is well known that the DOP is dependent on not only the light source but also the distance traveled by the optical signal in long haul network transmissions.

Since the noises at the upper and lower PDs are independent, the power of the noise sources in the output photocurrent can be written as

$$
\left\langle I_{\mathrm{PIIN}}^{2}\right\rangle=B R^{2} E\left[\int_{0}^{\infty} G_{0}^{2}(\nu, t) d \nu+\int_{0}^{\infty} G_{1}^{2}(\nu, t) d \nu\right] .
$$

The power of the differential-phase system's PIIN which exists in the photocurrent of the decoder is given by

$$
\left\langle I_{\mathrm{PIIN}}^{2}\right\rangle=\frac{B R^{2} P_{s r}^{2} K(K+3)}{4 \Delta \nu\left(P^{2}+1\right)} .
$$

The SAC-OCDMA systems with flat PSD of light sources in the coded bandwidth, performance is mainly limited by PIIN [6-11] due to light from the incoherent sources interfering at the PDs especially when the received power is large.

Finally, the CNR due to the effect of PIIN for conventional single-phase and the proposed differential-phase modulation schemes can be calculated as follows:

$$
\begin{aligned}
& \mathrm{CNR}_{\text {Single-phase }}=\frac{\left(I_{1}-I_{2}\right)^{2}}{\sigma_{\text {PIIN }}^{2}}=\frac{\Delta \nu}{6 K(K+1)\left(P^{2}+1\right) B}, \\
& \mathrm{CNR}_{\text {Differential-phase }}=\frac{\left(I_{1}-I_{2}\right)^{2}}{\sigma_{\text {PIIN }}^{2}}=\frac{\Delta \nu}{K(K+3)\left(P^{2}+1\right) B},
\end{aligned}
$$

where $I_{1}$ and $I_{2}$ are two average photocurrents of the upper photodiode and lower photodiode at the CS decoder.

Figure 3 plots the variation of the CINR with the number of simultaneous RBSs as a function of the length of several codes. It is clear that CNR performance of differential-phase 


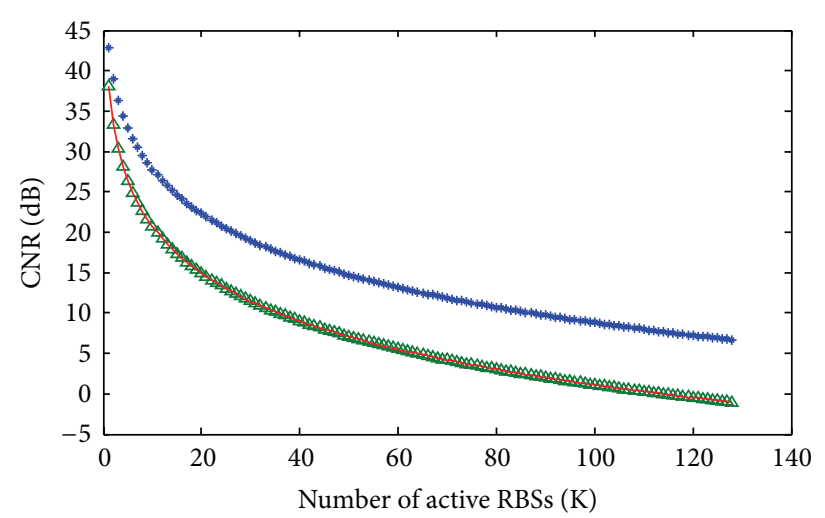

* Differential-phase (Hadamard) — Single-phase (Hadamard) $\Delta$ Single-phase (M-sequence)

FIGURE 3: CNR versus number of active RBSs for different code families.

SAC-OCDMA with CWH code is superior to conventional single-phase SAC-OCDMA with Hadamard code and Msequence code about $7.7 \mathrm{~dB}$ in large active RBSs scenario. The parameters of incoherent broadband sources used here have linewidth $60 \mathrm{~nm}$, center wavelength $1550 \mathrm{~nm}$, and the noiseequivalent electrical bandwidth of the receiver $B=80 \mathrm{MHz}$ (for the bit rate $155 \mathrm{Mb} / \mathrm{s}$ ).

The CNR of the conventional single-phase system degrades more significantly than that of the proposed differentialphase system, particularly with a large number of RBSs. The reason for this is that the PIIN effect becomes much larger since in the SAC-based OCDMA system, when a large number of RBSs transmit their coding patterns simultaneously, more wavelengths beat together during the direct detection by the square-law PDs. Besides, the performance in SACOCDMA system cannot be improved by increasing code length.

A common unpolarized amplified spontaneous emission (ASE) source can be used in the current differential-phase system because the scheme considers only the source power but not phase or polarization. However, on the long-haul transmissions over RoF network, the DOP effect must be addressed. In general, CNR can be improved by positioning a scrambler in front of balanced photodetector to eliminate the polarization-dependent effect of the detector. The scrambler theoretically removes the polarization sensitivity of the photodetector in the proposed RoF scheme; hence the average values of $s_{1}, s_{2}$, and $s_{3}$ in (12) approach zero, and the DOP is significantly decreased. In other words, the scrambler theoretically removes the polarization sensitivity of the photodetector in the proposed RoF scheme. In order to analyze the BER performance with variance of DOP (i.e., the DOP varies in the range 0 to 1 ) was assumed to represent the influence of polarization following long haul transmission.

As is shown in Figure 4, the BER performance of the proposed differential-phase scheme is characterized by an

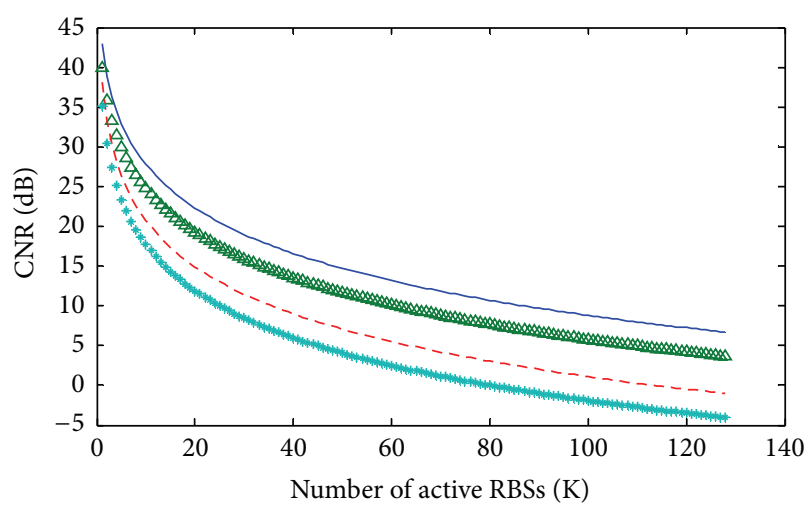

- Differential-phase $(\mathrm{DOP}=0)---$ Single-phase $(\mathrm{DOP}=0)$

$\triangle$ Differential-phase $(\mathrm{DOP}=1) \quad * \quad$ Single-phase $(\mathrm{DOP}=1)$

FIGURE 4: CNR performance with degree of polarization (DOP).

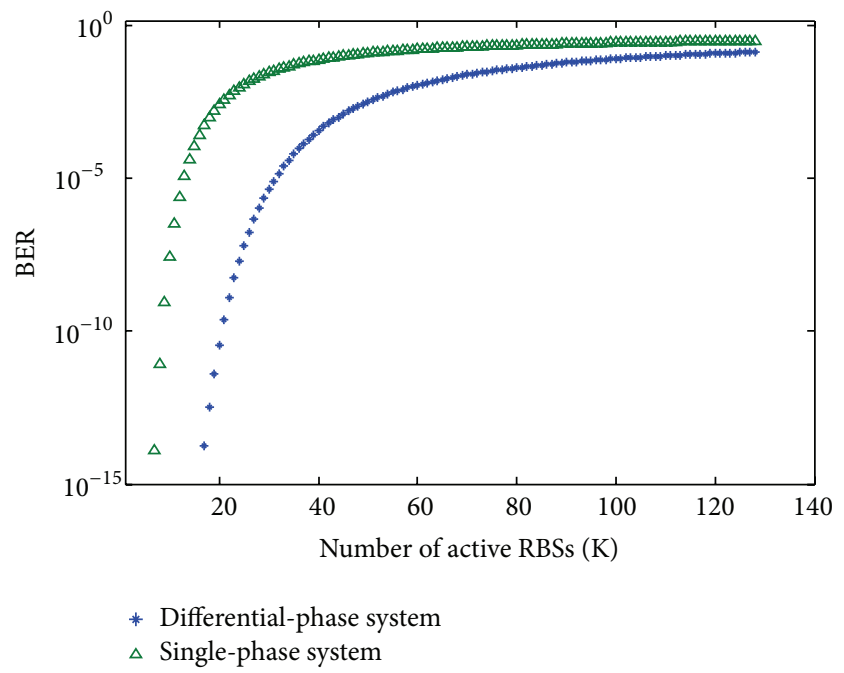

FIGURE 5: BER versus number of active RBSs for different modulation schemes.

upper bound of $P=1$ for the worst case and a lower bound of $P=0$ for the ideal case with the same assumption of Figure 3 . Compared to the average DOP of 1 for the worst case, the CNR of the proposed differential-phase scheme is improved about $7.7 \mathrm{~dB}$ when the number of RBSs is 120 . We can also find that the proposed differential-phase system even in high SOP condition is still performing better performance than singlephase system in the transmission links.

By assuming all the interference terms to be Gaussian distributed, the conditional BER can be calculated from amplitude shift keying (ASK) modulation; that is, on employing $\mathrm{BER}=0.5 \operatorname{erfc}(\sqrt{\mathrm{CINR} / 8})$, we can obtain the relation BER and the number of active RBSs as a function of the length. Figure 5 plots the variation of the BER with the number of active RBSs as a function of the length of differential-/singlephase systems. It can be seen that the BER of the conventional OCDMA network using single-phase scheme is worse than that of the differential-phase technology particularly in a large number of RBSs conditions. The reason for this is that 
when a large number of RBSs transmit their coding patterns simultaneously, more wavelengths beat together during the direct detection by the square-law PDs, the PIIN becomes the dominate noise degrading BER performance, and hence performance in SAC-OCDMA system cannot be improved by increasing code length of Hadamard codes. The BER performance of differential-phase scheme can support more than 19 active RBSs than single-phase scheme when the BER is $10^{-5}$. After BPF process, the transmitted signal power becomes the dominant issue to improve system performance.

\section{Conclusion}

The study proposes an AWG router-based OCDMA networks embedded with signal phase diversity scheme for RoF systems. The advantages of MAI and crosstalk beat noise effect in AWG routers can be suppressed by designed codec structure. In the case of ideal system constructed by the flattened source, each RBS requires only two AWG routers for spectral encoding and decoding processes; thus filter mismatch between network coders and decoders can be mitigated. Also, unlike FBG-based system, AWG router scheme exhibits no roundtrip time delay problem. Besides, AWG router-based codecs lies in that it has no accumulation of insertion loss when the total number of RBSs is increased. The CNR and BER of the proposed system are numerically analyzed by taking the dominate noise of PIIN into account. The result shows that the CNR of the proposed differential-phase system is superior $7 \mathrm{~dB}$ than other conventional single-phase OCDMA schemes in RoF system. The tradeoff on complementary codes in the study between system complexity and performance can be considered in different RoF links; hence the system flexibility is increased. In conclusion, the proposed system achieves a higher performance than a conventional RoF OCDMA scheme and can be implemented using a simple configuration comprising conventional low cost BLSs and compact optical components, rendering the overall system both cheap and straightforward.

\section{Acknowledgment}

This work was supported in part by the National Science Council under Grant no. NSC-102-2221-E-150-002.

\section{References}

[1] Z. Ahmed, D. Novak, R. B. Waterhouse, and H.-F. Liu, "37-GHz fiber-wireless system for distribution of broad-band signals," IEEE Transactions on Microwave Theory and Techniques, vol. 45, no. 8, pp. 1431-1435, 1997.

[2] C. T. Yen, H. C. Cheng, and I. J. Ding, "Hybrid analog/digital wavelength-time optical CDMA systems in radio-over-fiber transmissions," The Journal of Supercomputing, 2013.

[3] C. T. Yen, H. C. Cheng, Y. T. Chang, and W. B. Chen, "Performance analysis of dual unipolar/bipolar spectral code in optical CDMA systems," Journal of Applied Reserch and Technology, vol. 11, no. 2, pp. 235-241, 2013.

[4] B. K. Kim, S. Park, Y. Yeon, and B. W. Kim, "Radio-over-fiber system using fiber-grating-based optical CDMA with modified
PN codes," IEEE Photonics Technology Letters, vol. 15, no. 10, pp. 1485-1487, 2003.

[5] C. T. Yen, "Optical code-division multiple-access embedded with a polarisation diversity scheme for radio-over-fibre transmissions," IET Optoelectronics, vol. 6, no. 3, pp. 131-139, 2012.

[6] Z. Wei, H. M. H. Shalaby, and H. Ghafouri-Shiraz, "Modified quadratic congruence codes for fiber Bragg-grating-based spectral-amplitude-coding optical CDMA systems," Journal of Lightwave Technology, vol. 19, no. 9, pp. 1274-1281, 2001.

[7] J. F. Huang and D. Z. Hsu, "Fiber-grating-based optical CDMA spectral coding with nearly orthogonal M-sequence codes," IEEE Photonics Technology Letters, vol. 12, no. 9, pp. 1252-1254, 2000.

[8] C. C. Yang, J. F. Huang, and S. P. Tseng, "Optical CDMA network codecs structured with M-sequence codes over waveguidegrating routers," IEEE Photonics Technology Letters, vol. 16, no. 2, pp. 641-643, 2004.

[9] M. Kavehrad and D. Zaccarin, "Optical code-division-multiplexed systems based on spectral encoding of noncoherent sources," Journal of Lightwave Technology, vol. 13, no. 3, pp. 534-545, 1995.

[10] E. D. J. Smith, R. J. Blaikie, and D. P. Taylor, "Performance enhancement of spectral-amplitude-coding optical CDMA using pulse-position modulation," IEEE Transactions on Communications, vol. 46, no. 9, pp. 1176-1185, 1998.

[11] J.-F. Huang, C.-C. Yang, and S.-P. Tseng, "Complementary Walsh-Hadamard coded optical CDMA coder/decoders structured over arrayed-waveguide grating routers," Optics Communications, vol. 229, no. 1-6, pp. 241-248, 2004.

[12] Y. T. Chang, J. F. Huang, L. W. Chou, C. C. Wang, C. T. Yen, and H. C. Cheng, "Adaptive modified time-spreading and wavelength-group-hopping embedded M-sequence code for improved confidentiality over synchronous networks," Optical Engineering, vol. 50, no. 5, Article ID 055001, 2011.

[13] H. Takahashi, K. Oda, and H. Toba, "Impact of crosstalk in an arrayed-waveguide multiplexer on $\mathrm{N} \times \mathrm{N}$ optical interconnection," Journal of Lightwave Technology, vol. 14, no. 6, pp. 10971105, 1996.

[14] D. Castleford, A. Nirmalathas, D. Novak, and R. S. Tucker, "Optical crosstalk in fiber-radio WDM networks," IEEE Transactions on Microwave Theory and Techniques, vol. 49, no. 10, pp. 2030-2035, 2001.

[15] H. Takahashi, K. Oda, H. Toba, and Y. Inoue, "Transmission characteristics of arrayed waveguide $\mathrm{N} \times \mathrm{N}$ wavelength multiplexer," Journal of Lightwave Technology, vol. 13, no. 3, pp. 447455, 1995. 


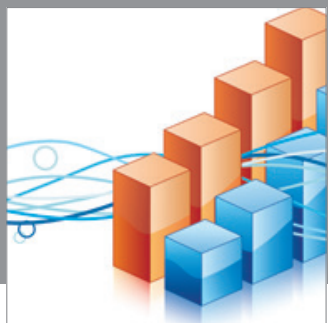

Advances in

Operations Research

mansans

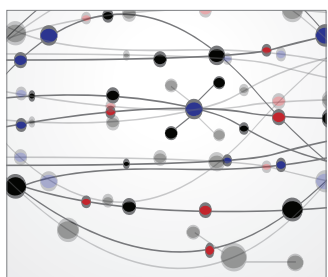

The Scientific World Journal
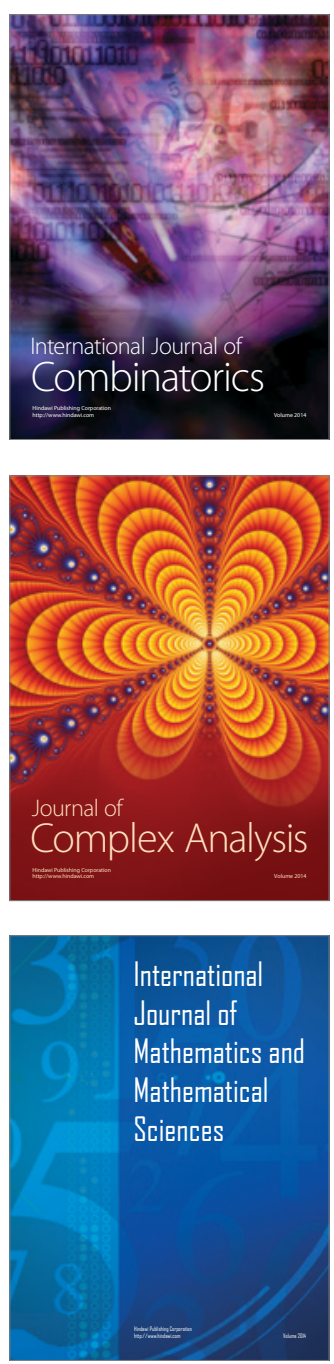
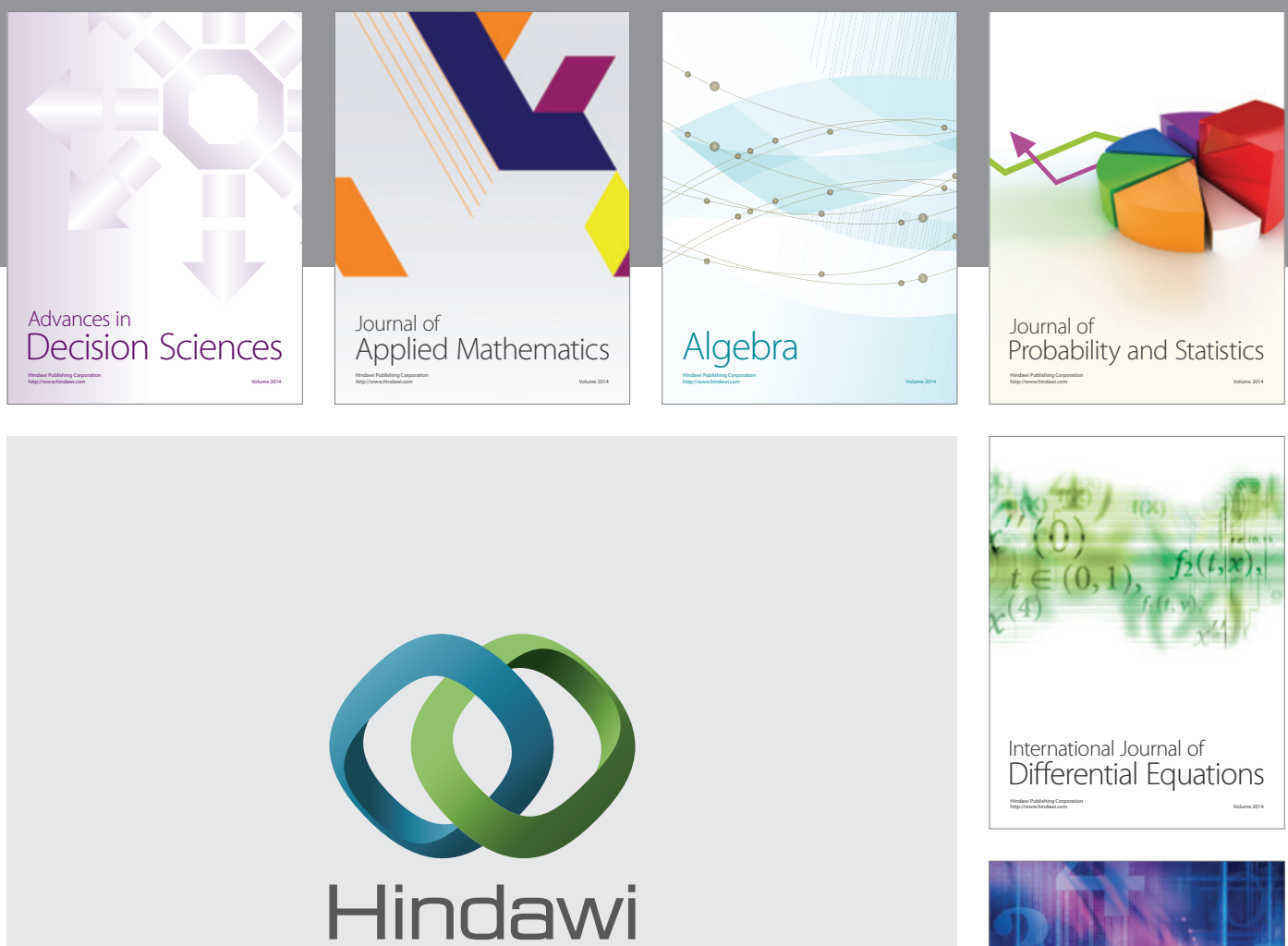

Submit your manuscripts at http://www.hindawi.com


Journal of

Function Spaces

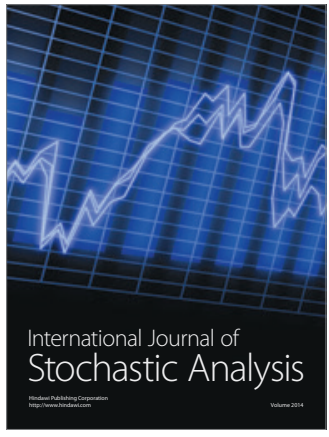

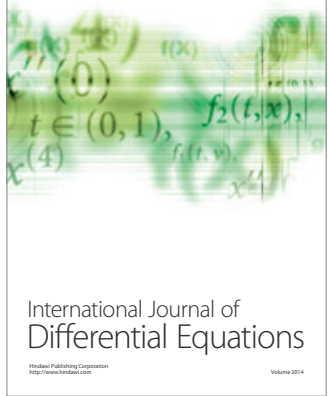
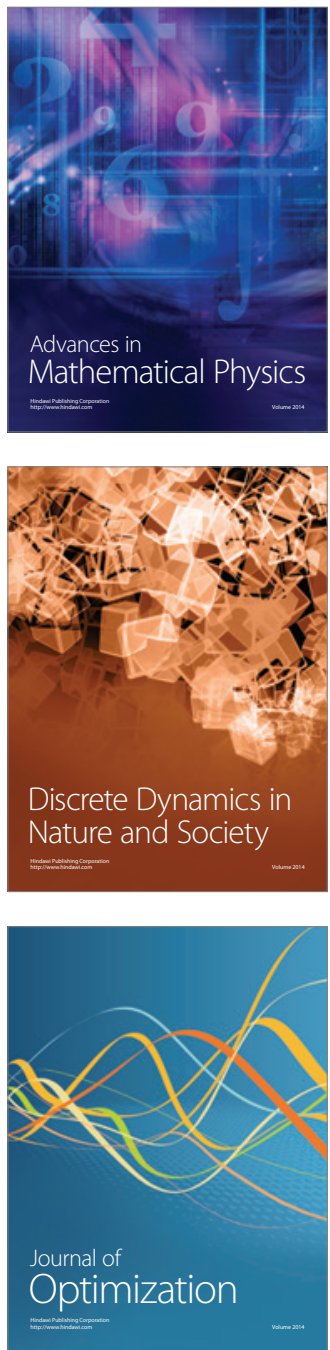